\title{
In Conversation: Evolution of Sports Medicine and Support for the Elite Indian Athlete-From 1960 to 2020
}

\author{
Mandeep S Dhillon ${ }^{1}$, Milkha Singh ${ }^{2}$, Jeev Milkha Singh ${ }^{3}$
}

\begin{abstract}
Milkha Singh is perhaps the most iconic track athlete from India, and his son Jeev Milkha Singh has been Asia's top Golfer for over 20 years. In conversation with the Editor MS Dhillon, the elite duo describe the level of medical support and the evolution of sports sciences relevant to elite athletes in the 1960s and compare it with the medical facilities and support that is available for athletes in India in the 21st century. Both discuss the issues that they feel are pertinent to develop sports sciences in India to a level equivalent to that in the world.

Keywords: Jeev Milkha Singh, Milkha Singh, Sports medicine, Sports sciences, Sports training.

Journal of Postgraduate Medicine, Education and Research (2020): 10.5005/jp-journals-10028-1415
\end{abstract}

Milkha Singh has for long been one of the icons of sports in independent India and has been the first to garner international fame in an individual sport; this was the era when the only sporting glory was brought to India by the field hockey team. He has inspired at least three generations of Indian athletes who have been awestruck with his training regimens and single-minded devotion to his sport, where he combined his grit and determination with his outstanding talent to beat the best in the world. For the last 50 years, he has been actively campaigning for the upgradation of facilities for training, coaching, and rehabilitation of athletes. His son Jeev Milkha Singh is an icon in his own right; he single-handedly imprinted the game of Golf, previously regarded as the domain of the rich for networking and leisure, onto the mind of the average Indian. For many years, he has remained the premier golfer in Asia, winning on the European and Asian tours, and has become a source of inspiration to many of the golfing names that have emerged in the 21st century.

In a candid conversation with JPMER Editor Prof MS Dhillon, the two elite athletes discuss the state of affairs for medical and administrative support to the elite athlete back in the 1960s, when Milkha Singh was at his peak and compare it with the modern era in 2020, when Jeev Milka Singh is at his peak.

Dhillon: Milkha Singh ji, when you were running at the peak of your prowess, in the 1960s, what was the support systems in place for you with regard to the medical facilities and physiotherapy?

Milkha Singh: I was at my peak from 1956 to 1964 , and at that time, there was never any doctor or medical personnel associated with any team. I remember getting a "pulled muscle" while training in Patiala; there was a doctor whom I consulted who said he would inject me, and I would get better. But my coach Ranbir Singh, and another German coach, stopped me from that, saying that I should get better using natural methods only, by continuing exercises, etc. I got the injection but got no improvement; the German coach then told me to focus on exercises and jogging and said that I would get better after 2 to 3 weeks and that is what happened. I carried on doing low-grade exercises and slowly got better.

Dhillon: Do you think that in the 1960s, even the doctors were not fully aware of the problems of athletes and how to manage them?

Milkha Singh: Nobody knew, not the doctors, not the coaches, and not even the athletes themselves. Nobody really knew much about injury prevention and appropriate rehabilitation.
${ }^{1}$ Department of Orthopaedics, Postgraduate Institute of Medical Education and Research, Chandigarh, India

${ }^{2}$ Olympic Sprint athletics, Chandigarh, India

${ }^{3}$ International Golf, Chandigarh, India

Corresponding Author: Mandeep S Dhillon, Department of Orthopaedics, Postgraduate Institute of Medical Education and Research, Chandigarh, India, Phone: +91 9815951090, e-mail: drdhillon@gmail.com

How to cite this article: Dhillon MS, Singh M, Milkha Singh J. In Conversation: Evolution of Sports Medicine and Support for the Elite Indian Athlete-From 1960 to 2020. J Postgrad Med Edu Res 2020;54(4):175-177.

Source of support: Nil

Conflict of interest: None

Dhillon: The same questions to you Jeev. When you entered the international arena as a top Golfer, in the mid-1990s, what do you think were the medical support facilities that were available to the elite and the average athlete, and are they any different now in 2020?

Jeev Milkha Singh: When I started, although things had changed, there was a lot of information available in the world; however, local support in India was minimal. We did not know anything about training, especially which muscles are important for my specific sport (Golf), and how I should focus my training to develop these. I would just jog to the Lake Club Gym and would do an overall body fitness and training protocol. I did not know that in Golf everything involves "pulling"; by that I mean that when in your swing phase, when you take your clubs to the back, you have to "pull" them forward using a specific set of muscles. For this, rowing is actually a very good exercise, and none of the golfers knew this, nor did anyone tell me; and this made a difference in the incidence of getting injuries as well as recovering from them. As an amateur on the golfing circuit, we had no physios, nor any guidance; when we became professionals, the international circuit had trained physios who used to tell us and guide us about which muscles to "stabilize and fix" and which ones to stretch, etc. We were also made aware about nutrition; we never knew adequately about things like that on the golf course you need sufficient water and amino acids in your body so that your muscles do not cramp. 
Dhillon: That is true and very relevant; today, we have specialized sports physicians who look at all these aspects, and it is not left to orthopedic surgeons to manage! My practice of sports medicine and knowledge has also evolved in the last 25 years; when I started arthroscopy and looking after sportspersons, we had to guide them about everything from doing surgery, to rehab, and even diet and often warn them about banned drugs! Do you think that even in your 25 years of International Golf, there has been an evolution in the field of medicine wherein support systems for athletes have changed?

Jeev Milkha Singh: For Sure! I think if I had known when I started golf which muscles I have to strengthen, and which to stretch, what I need to eat on the course and before my game, etc., I would not have been injured so many times!

Dhillon: Another question addressed to both of you as you represent two different eras. Do you think that in the 1960s and in 2000s there was a difference in the facilities available to Indian sportspersons when compared to the world?

Milkha Singh: A big difference! Even in the 1960s, international athletes trained on scientific lines, while we in India had no knowledge or information. Like, I used to run 400 meters, and the race needs 3 to 4 issues that need to be understood. One you need stamina, two you need speed, and 3 you need strength; if these three to four things are not combined in the right way, you cannot adequately compete. My coaches used to put a bench and I would do exercises for the upper limb by bench presses; we never had any gyms as you have today. In modern times, sprinters work on leg muscles, work on abdomen and hip muscles, and even arms, all in a coordinated way. In my time, weight and endurance training meant that I picked up my co-athlete on my shoulders and started running! This and bench presses were the main exercises we used to do; however, we used to improvise and run up and down hills as well as run barefoot in sand, which allowed us to run very fast on regular grounds. But in other countries, they had more scientific programs that allowed them to develop their bodies better.

Dhillon: A question to you Jeev. Things have probably changed in the 21st century. Do you think that a young guy from India, who comes onto the professional tour today, would have come from a training background that is much better and of International standards, or is there still a difference when compared to the rest of the world?

Jeev Milkha Singh: Well, I would stay that even today there is a difference, but it is only 15 to $20 \%$. One reason is that all information is available on the Internet. The youngsters are much more aware, and they model their schedules on that of the top golfers of the world. They google him, check his schedules, his gym protocols, even his diet patterns, and model their training protocols on to that. They follow his nutrition and mental strengthening patterns so as not to lose concentration in the last few holes of golf, which is critical for success.

Dhillon: Well I would like to tell you that at PGI we have had a Sports Medicine Clinic focusing on many aspects that you have mentioned, which has been functioning for more than 15 years. Additionally, there are at least three Medical Schools in India, including one in the army, who have started focused MDs in sports medicine. These guys are not physios, not orthopedic surgeons but just physicians trained in exactly what you mention and help in upgrading preparation of athletes and focus on injury prevention.

Jeev Milkha Singh: Great news. I think this will help the whole sporting fraternity in India to come up to international levels. Small things related to performance are what makes an athlete win or lose at a crucial time. I never used to eat nuts, bananas, etc. on the course. Now l eat 3 bananas and lots of nuts due to nutritional advice, and I am better for it. Twenty years ago, when I used to walk off a golf course, I was always so hungry; now I am not, meaning that my nutrition levels and blood sugars have been kept maintained, which is reflected in my game too.

Milkha Singh: I would like to add something about the difference of medical and coaching support between the two eras. We were three athletes in my time, Dhyan Chand in Hockey, Lala Amarnath in Cricket, and Milkha Singh in athletics. We had no formal coaches or trainers; all coaches in India have been produced after the 1960s. I had no formal coach, and the person who coached me was a physical instructor in a school. It was only when the National Institute of Sports was opened at Patiala that formal training for coaches was started. Today, in India, there are at least 50,000 trained coaches; but the result in athletics, etc. is still zero. Look at the great runners from India, PT Usha, Anju George, Milkha Singh, all were self-made. My grouse against the modern coaches, at least of the 1980 s and 1990s, is that many have tried to enhance performance by using drugs, etc. There is a role of some doctors also who help in temporarily enhancing performance at the state and national level, which is to be condemned. On the other hand, we have a lot of coaches today who have helped athletes; Gopichand has helped badminton to a great deal, Boxing coaches have done wonders, and wrestling from Haryana has improved to a great extent due to excellent coaching. But still a large majority of coaches are not actually doing their job and are just getting paid. They should get on to the field and play alongside their wards and get physically involved and not sit on the sidelines.

Jeev Milkha Singh: Well one of the issues could be that many of the coaches may not actually be understanding what is involved in their job profile. We all evolve with time and educate ourselves. Continuing education to stay abreast of modern methods is very important; I meet a lot of youngsters on the golf course who talk about newer things, methods, and equipment. I immediately read about it on the Internet and try to evolve to stay up to date. This is what is needed for all the coaching staff and support personnel in India.

Milkha Singh: Let me tell you one thing; today, it is extremely difficult for India to win an individual Olympic medal in track and field sports. Olympics are not like Cricket where only 10 to 15 countries play, and you win some and you lose some. A total of 230 countries participate in the Olympics, and unless there is a coordinated effort, individual performance will fail. I met Gopichand in Mumbai recently and asked him how he produces such good level of players consistently. He told me that before the kids come for coaching, he spends a lot of time creating a special training protocol for each player, one night before. He makes the effort of identifying strengths and weaknesses of all players his trainees play against and then plans and mentors them how to counter them. You cannot win medals by coaches sitting on the sidelines.

Dhillon: So what I gather form both of you, along with my understanding, is that even in 2020, the support staff of the athletes needs to be brought to an international level. There has to be a team of nutritionist, mental strengthening, physio, trainer, etc. along with the coach which will enable our athletes to develop.

Jeev Milkha Singh: But on the other hand, there has been a great change in medical support as well as the equipment available, along with change in mind-set of athlete and medical staff. 
Milkha Singh: A Big difference! When I started running in 1951, I was running barefoot; till 1956, we in India never knew what running shoes were. The first time I wore shoes for running was in 1956 Olympics. Another thing. The running shoes of my time were hard leather, at least $1 \mathrm{~kg}$ or more weight, with hard plates in them and heavy metal spikes. The shoes today feel like they are made of paper!

Jeev Milkha Singh: Now you can order shoes according to your individual foot and biomechanics. They take your footprint and make a shoe individualized for you alone; What a change even during my career of 25 years!

Milkha Singh: Change in attitude and availability of equipment both are important; many athletes today desire to win but often do not put in the required effort, although they think they are doing their best. I used to run 400-meters race many times a day, and my coach would say do it again! I would often vomit blood, but we would still continue to practice and that too with no regard to the weather. Dhyan Chand was also a self-taught man; I know he used to tie a cycle tire in a goal post and try and push 500 balls into the goal from the sides of that, and all on his own. My coach used to make me do double the practice of any other athlete because he saw the potential in me, and I supported him with just my will power.

Dhillon: Thank you sirs, both you Milkhas Singh ji and you Jeev have been iconic sportspersons from India and have inspired generations of budding sportspersons. This interview definitely sheds light on the personal effort an athlete has to make as well the deficiencies in the support personnel and facility that are still persisting in our country in 2020. A lot has changed over 50 years, but it seems that a lot more is needed before India can compete with the world.

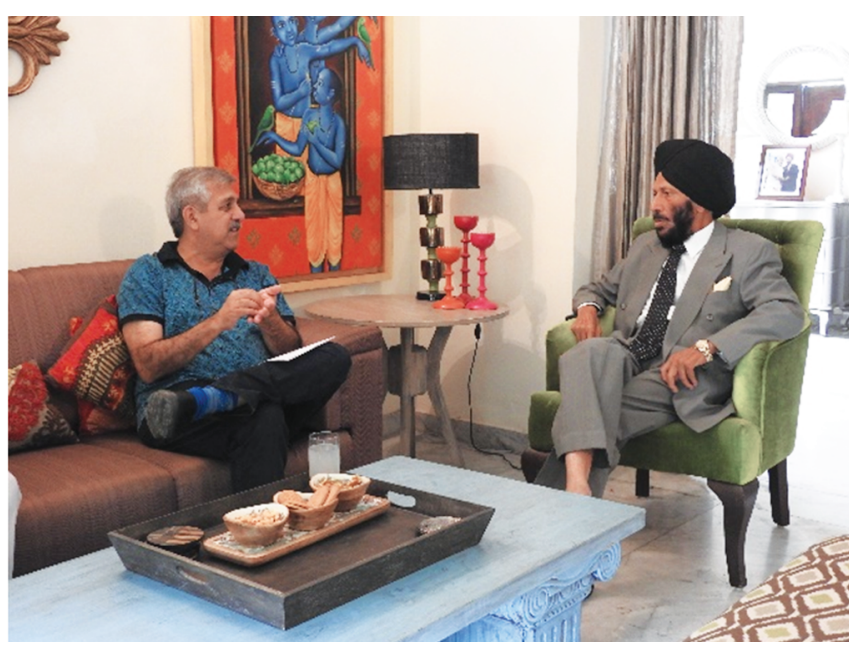

\section{Recommended Further Reading}

1. https://www.olympicchannel.com/en/athletes/detail/milkha-singh/.

2. https://scroll.in/field/960240/pause-rewind-play-when-milkhasingh-created-history-and-clinched-indias-first-commonwealthgold.

3. https://www.pgatour.com/players/player.20686.jeev-milkha-singh. html.

4. https://indianexpress.com/article/sports/golf/jeev-milkha-singhthe-grateful-golfer-6070679/.
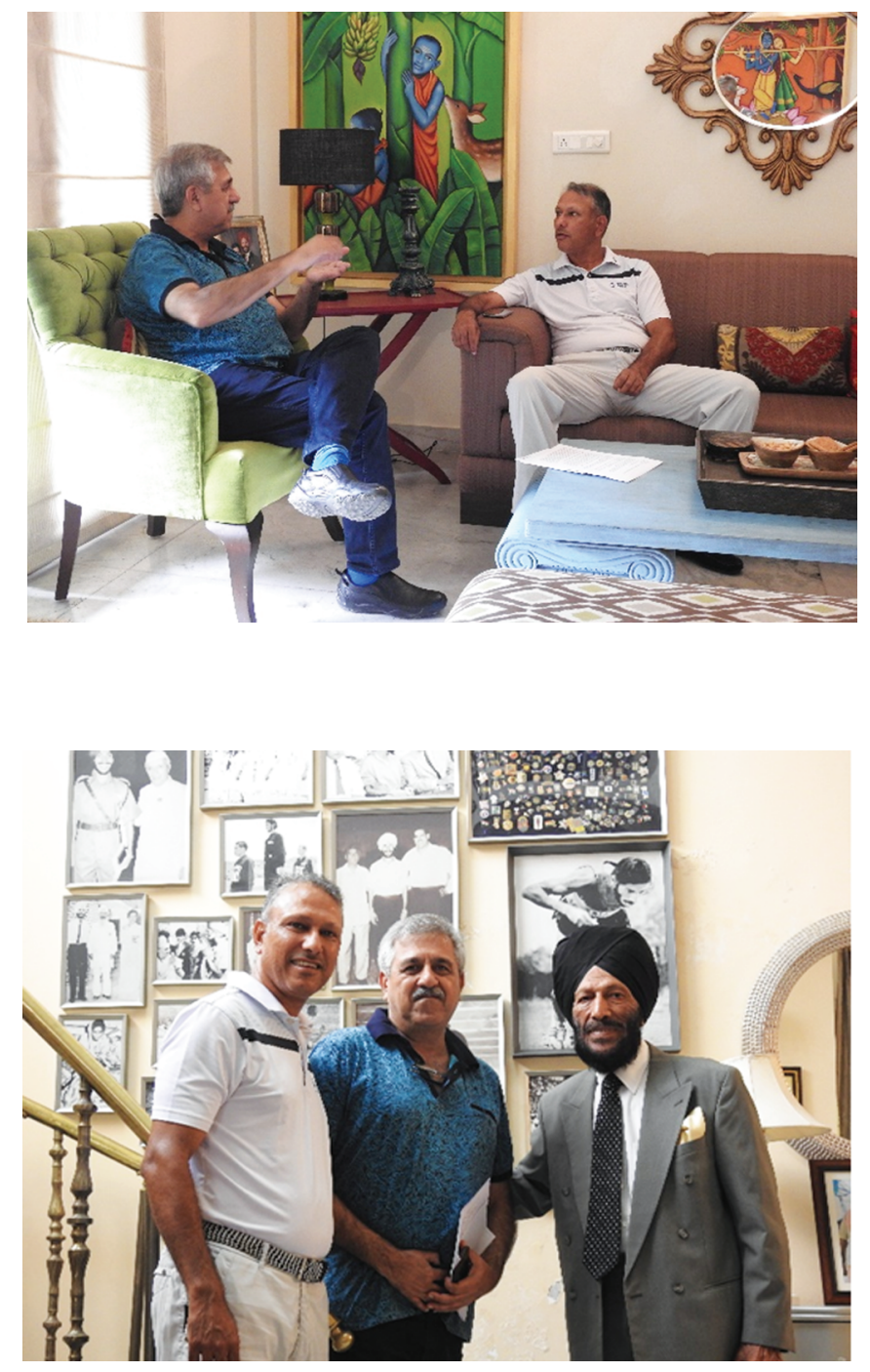